\title{
Internal structure and stability of vortices in a dipolar spinor Bose-Einstein condensate
}

\author{
Magnus O. Borgh, ${ }^{1,2}$ Justin Lovegrove, ${ }^{2}$ and Janne Ruostekoski ${ }^{2}$ \\ ${ }^{1}$ Faculty of Science, University of East Anglia, Norwich, NR4 7TJ, United Kingdom \\ ${ }^{2}$ Mathematical Sciences, University of Southampton, Southampton, SO17 1BJ, United Kingdom
}

(Dated: April 6, 2017)

\begin{abstract}
We demonstrate how dipolar interactions can have pronounced effects on the structure of vortices in atomic spinor Bose-Einstein condensates and illustrate generic physical principles that apply across dipolar spinor systems. We then find and analyze the cores of singular vortices with nonAbelian charges in the point-group symmetry of a spin- $3{ }^{52} \mathrm{Cr}$ condensate. Using a simpler model system, we analyze the underlying dipolar physics and show how a characteristic length scale arising from the magnetic dipolar coupling interacts with the hierarchy of healing lengths of the $s$-wave scattering, and leads to simple criteria for the core structure: When the interactions both energetically favor the ground-state spin condition, such as in the spin-1 ferromagnetic phase, the size of singular vortices is restricted to the shorter spin-dependent healing length. Conversely, when the interactions compete (e.g., in the spin-1 polar phase), we find that the core of a singular vortex is enlarged by increasing dipolar coupling. We further demonstrate how the spin-alignment arising from the interaction anisotropy is manifest in the appearance of a ground-state spin-vortex line that is oriented perpendicularly to the condensate axis of rotation, as well as in potentially observable internal core spin textures. We also explain how it leads to interaction-dependent angular momentum in nonsingular vortices as a result of competition with rotation-induced spin ordering. When the anisotropy is modified by a strong magnetic field, we show how it gives rise to a symmetry-breaking deformation of a vortex core into a spin-domain wall.
\end{abstract}

\section{INTRODUCTION}

The achievement of Bose-Einstein condensation using atoms with large magnetic dipole moments, such as ${ }^{52} \mathrm{Cr}\left[1[3],{ }^{168} \operatorname{Er}[4]\right.$, and several Dy isotopes [5, [6] as well as creation of a degenerate dipolar Fermi gas [7] have opened up a new avenue for studying the effects of long-range and anisotropic interactions in ultracold atomic gases [8]. In such systems, long-range magnetic order can coexist with superfluidity, making possible, e.g., ferro-superfluids [9]. The interaction can then lead to novel instabilities, e.g., toward formation of droplet crystals [10 12], and formation of a condensate may be strongly influenced by the spin dynamics 13 . The interaction can also profoundly affect the stability and structure of vortices [14 18], e.g., inducing a phase transition from straight to twisted vortex lines [19]. Simultaneously, the structure of topological defects and textures is a central topic in the study of spinor Bose-Einstein condensates (BECs), where the atomic spin degree of freedom is not frozen out by strong magnetic fields [20]. This gives rise to a rich phenomenology of the internal structure of vortices 2132 . Recent experiments have demonstrated controlled preparation of nonsingular vortices $33-36$ as well as point defects [37, 38] and particle-like solitons [39]. The in situ observation of splitting of singly quantized vortices into pairs of half-quantum vortices [40], theoretically predicted in Ref. [27], marks an increasing experimental interest in the internal core structure.

The spin degree of freedom in spinor BECs also implies that dipolar interactions (DIs) arising from the magnetic dipole moment of the atoms can have a strong impact on the spin texture [41 43]. Nontrivial textures arising spontaneously due to DI have been observed in experiment [44, 45]. However, the potentially large impact on the internal structure of vortices has so far been little studied. Even a very weak DI can influence the relaxation of vortices by changing the longitudinal magnetization, whose conservation in $s$-wave scattering can be important for stability and structure, e.g., of a coreless vortex in a polar condensate [30, 31]. Theoretical works on vortices in dipolar spinor BECs have predicted a superfluid Einstein-de Haas effect, where magnetic relaxation induces vortex formation [46-49], as well as a stable spin vortex in a nonrotating system [50-52]. In a rotating highly oblate condensate, complex multivortex states and stable higher-order defects have been described [53].

Here we demonstrate how dipolar interactions can have pronounced effects on the internal structure of vortices in atomic spinor BECs. To clearly illustrate the underlying physical principles, we employ the comparative simplicity of a spin-1 model system. The dipolar effects arise from generic properties of the DI and spinor systems and the corresponding principles may therefore be applied more broadly to understand properties of vortices in dipolar spinor BECs. Dipolar spin-1 BECs could potentially also be realized using alkali-metal atoms by suppressing the $s$-wave scattering lengths via optical or microwave Feshbach resonances [54, 55]. As an example of experimentally realized dipolar BEC, we numerically find and analyze the stable core structure of a singular vortex in a spin-3 condensate of ${ }^{52} \mathrm{Cr}$. The ${ }^{52} \mathrm{Cr}$ atom possesses a relatively large magnetic dipole moment [46] and is predicted to exhibit a dihedral-6 pointgroup order-parameter symmetry in the ground state, supporting non-Abelian vortices. 
The multicomponent condensate wave function of a spinor BEC allows the condensate to maintain the superfluid density in the core of singular vortices. In addition to depleting the condensate density, the wave function can also be excited out of the ground-state manifold to accommodate the order-parameter singularity and form a filled defect core. Here we numerically find the superfluid cores of singular vortices when the atoms exhibit a long-range and anisotropic magnetic DI.

The DI gives rise to a new spin-dependent healing length, adding to the hierarchy of characteristic length scales arising form the contact interaction to determine the structure of singular-vortex cores [27, 32, 56]. We analyze the interplay of these length scales and demonstrate how the size of a singular-vortex core is determined by the shorter of the spin-dependent healing lengths when the DI and contact interaction both restrict breaking of the ground-state spin condition (the ground-state phase of the bulk superfluid), e.g., in the ferromagnetic (FM) spin-1 BEC. On the other hand, when the contact interaction and DI compete, such as in the spin-1 polar phase, we explain how a singular-vortex core expands with increasing DI, beyond the size in its absence. In addition, the anisotropy of the interaction leads to an internal spin texture that is potentially observable in a spin- $3{ }^{52} \mathrm{Cr}$ condensate.

We further analyze manifestations of the interaction anisotropy in the spin-1 model system and and show it leads to a ground-state spin vortex that appears perpendicularly to the axis of a slow rotation. The structure is then the result of interplay between dipolar spin alignment and rotation as the vortex line bends to adapt to the latter. At more rapid rotation, we demonstrate a nontrivial interaction dependence of the angular momentum carried by a ground-state coreless vortex. We show how it may be understood from a competition between dipolar spin alignment and the adaptation of the spin texture to rotation.

Drastically different spin-ordering effects can appear in the presence of a sufficiently strong external magnetic field, such that the DI may be averaged over the rapid spin precession [41]. We show how the resulting modified interaction anisotropy leads to a symmetry-breaking core deformation with increasing DI in a stable singular vortex. At sufficiently strong DI, the vortex core deforms into a domain wall separating regions with opposite spin polarization.

\section{MEAN-FIELD THEORY OF THE DIPOLAR BEC}

We treat the spinor BEC in the classical GrossPitaevskii mean-field theory, which can be straightforwardly extended to include DI between the atoms. Here we first give a brief overview of the salient points (for full details see, e.g., Ref. 20]) in the spin-1 case, and then show how the theory is modified for spin- 3 atoms.

\section{A. Spin-1}

The spin-1 condensate wave function $\Psi$ may be expressed in terms of the atomic density and a normalized three-component spinor as

$$
\Psi(\mathbf{r})=\sqrt{n(\mathbf{r})} \zeta(\mathbf{r})=\sqrt{n(\mathbf{r})}\left(\begin{array}{c}
\zeta_{+} \\
\zeta_{0} \\
\zeta_{-}
\end{array}\right), \quad \zeta^{\dagger} \zeta=1 .
$$

The expectation value $\langle\hat{\mathbf{F}}\rangle=\zeta_{\alpha}^{\dagger} \hat{\mathbf{F}}_{\alpha \beta} \zeta_{\beta}$ of the spin operator, defined as the vector of spin-1 Pauli matrices, gives the condensate spin. This relates to the magnetic dipole moment arising from the intrinsic angular momentum of the atom as $\mathbf{m}=-g_{F} \mu_{\mathrm{B}}\langle\hat{\mathbf{F}}\rangle$ [57], where $g_{F}$ is the Landé factor and $\mu_{\mathrm{B}}$ is the Bohr magneton. The Hamiltonian density including the DI is then given by

$$
\mathcal{H}=h_{0}+\frac{c_{0}}{2} n^{2}+\frac{c_{2}}{2} n^{2}|\langle\hat{\mathbf{F}}\rangle|^{2}+\frac{c_{\mathrm{d}}}{2} \int D\left(\mathbf{r}, \mathbf{r}^{\prime}\right) d^{3} r^{\prime},
$$

where

$$
D\left(\mathbf{r}, \mathbf{r}^{\prime}\right)=\frac{\mathbf{F}(\mathbf{r}) \cdot \mathbf{F}\left(\mathbf{r}^{\prime}\right)-3[\mathbf{F}(\mathbf{r}) \cdot \hat{\mathbf{n}}]\left[\mathbf{F}\left(\mathbf{r}^{\prime}\right) \cdot \hat{\mathbf{n}}\right]}{\left|\mathbf{r}-\mathbf{r}^{\prime}\right|^{3}}
$$

describes the interaction of dipoles at $\mathbf{r}$ and $\mathbf{r}^{\prime}$ given by the local condensate spin with the coupling constant $c_{\mathrm{d}}=$ $\mu_{0} \mu_{\mathrm{B}}^{2} g_{F}^{2} /(4 \pi)$ [57], where $\mu_{0}$ is the vacuum permeability. We define $\mathbf{F}=n\langle\hat{\mathbf{F}}\rangle$ and denote the unit vector along $\mathbf{r}-\mathbf{r}^{\prime}$ by $\hat{\mathbf{n}}$. The single-particle Hamiltonian density

$$
h_{0}=\frac{\hbar^{2}}{2 M}|\nabla \Psi|^{2}+\frac{1}{2} M \omega^{2} r^{2} n,
$$

where $M$ is the atomic mass, includes the external trapping potential, which we take to be an isotropic harmonic oscillator with frequency $\omega$. The contactinteraction strengths are given by the scattering lengths $a_{f}$ in the spin- $f$ channel of colliding spin- 1 atoms as $c_{0}=4 \pi \hbar^{2}\left(2 a_{2}+a_{0}\right) /(3 M)+c_{0}^{\mathrm{d}}$ and $c_{2}=4 \pi \hbar^{2}\left(a_{2}-\right.$ $\left.a_{0}\right) /(3 M)+c_{2}^{\mathrm{d}}$. Here we have made it explicit that the coupling constants may be modified by contributions $c_{0,2}^{\mathrm{d}}$ arising from an absorbed contact-interaction part of the DI (see below and Appendix A).

The spin-1 BEC exhibits two ground state phases: a FM phase that maximizes the condensate spin $|\langle\hat{\mathbf{F}}\rangle|=1$ and a polar phase where $|\langle\hat{\mathbf{F}}\rangle|=0$ in a uniform system. Without DI, the ground-state phase is determined by the sign of $c_{2}$ with a negative value favoring the FM phase. When magnetic DI, where the dipole moment is proportional to the condensate spin, is present, the ground state depends also on $c_{\mathrm{d}}$. In particular, from Eq. (3) we can see that the DI is minimized when spins $|\langle\hat{\mathbf{F}}\rangle|=1$ are aligned head-to-tail. The DI will therefore also favor formation of a FM phase, and sufficiently large $c_{\mathrm{d}}$ may overcome also a positive $c_{2}$ [50].

From Eq. (2) the familiar coupled Gross-Pitaevskii equations (GPEs) describing the condensate dynamics 
may be derived. Following Ref. [20], we write the contribution from the DI term in the equation for the $\psi_{m}=$ $\sqrt{n} \zeta_{m}$ spinor component as

$$
i \hbar \frac{\partial \psi_{m}(\mathbf{r})}{\partial t}=\ldots+c_{\mathrm{d}} \sum_{j} \hat{\mathbf{F}}_{m j} \psi_{j}(\mathbf{r}) \cdot \mathcal{B}(\mathbf{r}) .
$$

The vector $\mathcal{B}$ is given by

$$
\mathcal{B}(\mathbf{r})=\int \frac{\mathbf{F}\left(\mathbf{r}^{\prime}\right)-3 \hat{\mathbf{n}}\left[\mathbf{F}\left(\mathbf{r}^{\prime}\right) \cdot \hat{\mathbf{n}}\right]}{\left|\mathbf{r}-\mathbf{r}^{\prime}\right|^{3}} d^{3} r^{\prime}
$$

and is related to the magnetic field

$$
\begin{aligned}
& \mathbf{B}(\mathbf{r})=\frac{\mu_{0}\left\{3 \hat{\mathbf{n}}\left[\mathbf{m}\left(\mathbf{r}^{\prime}\right) \cdot \hat{\mathbf{n}}\right]-\mathbf{m}\left(\mathbf{r}^{\prime}\right)\right\}}{4 \pi\left|\mathbf{r}-\mathbf{r}^{\prime}\right|^{3}}+\frac{2 \mu_{0}}{3} \mathbf{m}\left(\mathbf{r}^{\prime}\right) \delta\left(\mathbf{r}-\mathbf{r}^{\prime}\right) \\
& =-g_{F} \mu_{\mathrm{B}} \mu_{0}\left\{\frac{3 \hat{\mathbf{n}}\left[\mathbf{F}\left(\mathbf{r}^{\prime}\right) \cdot \hat{\mathbf{n}}\right]-\mathbf{F}\left(\mathbf{r}^{\prime}\right)}{\left|\mathbf{r}-\mathbf{r}^{\prime}\right|^{3}}+\frac{2}{3} \mathbf{F}\left(\mathbf{r}^{\prime}\right) \delta\left(\mathbf{r}-\mathbf{r}^{\prime}\right)\right\}
\end{aligned}
$$

at $\mathbf{r}$ arising from the condensate dipole moment at $\mathbf{r}^{\prime}$. The factor $g_{F} \mu_{\mathrm{B}} \mu_{0} /(4 \pi)$ enters the coupling constant $c_{\mathrm{d}}$ and the $\delta$-function term yields a contact-interaction contribution that is absorbed in $c_{0,2}$ as above (see also Appendix A). Integrating the remaining term over $\mathbf{r}^{\prime}$ yields $\mathcal{B}$.

The DI term in Eq. (5) is nonlocal, and its evaluation involves finding the integral over $\mathbf{r}^{\prime}$, which is computationally expensive. However, since the integral in Eq. (6) has the form of a convolution, it can be computed efficiently in Fourier space, where the convolution of two functions becomes a multiplication of their Fourier transforms. For our computations we follow the formalism of Ref. [20], rewriting $\mathcal{B}$ as

$$
\mathcal{B}_{\alpha}=-\sum_{\beta} \int \mathrm{Q}_{\alpha \beta}\left(\mathbf{r}-\mathbf{r}^{\prime}\right) F_{\beta}\left(\mathbf{r}^{\prime}\right) d^{3} r^{\prime},
$$

where the tensor $Q$ is defined as

$$
\mathrm{Q}_{\alpha \beta}(\mathbf{r})=\frac{3 \hat{r}_{\alpha} \hat{r}_{\beta}-\delta_{\alpha \beta}}{r^{3}},
$$

for $\hat{\mathbf{r}}=\mathbf{r} / r$. In Eq. (8) the convolution is explicit and Fourier transformation immediately gives

$$
\tilde{\mathcal{B}}_{\alpha}(\mathbf{k})=-\sum_{\beta} \tilde{\mathbf{Q}}_{\alpha \beta}(\mathbf{k}) \tilde{F}_{\beta}(\mathbf{k}) .
$$

To compute $\mathcal{B}$ we then need the Fourier transforms on the right-hand side, where $\tilde{\mathbf{F}}(\mathbf{k})$ must be found numerically, while $\tilde{Q}(\mathbf{k})$ can be found analytically as (see Appendix $\mathrm{A}$ and Ref. [20])

$$
\tilde{\mathbf{Q}}_{\alpha \beta}(\mathbf{k})=-\frac{4 \pi}{3}\left(3 \hat{k}_{\alpha} \hat{k}_{\beta}-\delta_{\alpha \beta}\right),
$$

where $\hat{\mathbf{k}}=\mathbf{k} / k$. Note that the derivation of this Fourier transform rests on nontrivial assumptions. We provide the details in the Appendix $\mathrm{A}$.
In practical numerical calculations using Fast Fourier Transforms, the long-range nature of the DI can lead to aliasing problems that yield erroneous results, and accuracy may more generally be reduced. These problems can be avoided or mitigated by truncating the dipolar interaction [58, 59]. In a spherical or nearly spherical system, where computations are performed on a grid with all sides equal, the simplest solution is to truncate the dipolar interaction at a radius $R$, such that $\mathrm{Q}(\mathbf{r})=0$ for $r>R$. The Fourier transform of the truncated interaction is then

$$
\begin{aligned}
& \tilde{\mathbf{Q}}_{\alpha \beta}^{r<R}(\mathbf{k})=\int_{r<R} \mathrm{Q}_{\alpha \beta}(\mathbf{r}) e^{-i \mathbf{k} \cdot \mathbf{r}} d^{3} r \\
& =-4 \pi\left(3 \hat{k}_{\alpha} \hat{k}_{\beta}-\delta_{\alpha \beta}\right)\left(\frac{1}{3}+\frac{k R \cos (k R)-\sin (k R)}{(k R)^{3}}\right),
\end{aligned}
$$

which is the spherical cut-off found in Ref. [58], straightforwardly generalized to the spinor case (see Appendix A).

In the presence of an external magnetic field $\mathbf{B}_{\text {ext }}=$ $B_{\text {ext }} \hat{\mathbf{z}}$, the condensate spin precesses with the Larmor frequency $\omega_{\mathrm{L}}=g_{F} \mu_{\mathrm{B}} B_{\text {ext }} / \hbar$. In a sufficiently strong field, the precession is rapid compared with the DI-induced spin dynamics. It is then convenient to describe the condensate in the spin-space frame rotating at the Larmor frequency through the transformation $\zeta_{m}(\mathbf{r}, t) \rightarrow$ $\zeta_{m}(\mathbf{r}, t) e^{-i m \omega_{\mathrm{L}} t}[41,42]$. This leaves all terms of Eq. (2) invariant, except the dipolar interaction. (Also the linear Zeeman term that would arise from the magnetic field is canceled and we assume any quadratic Zeeman energy to be small.) The modified dipolar interaction is found as a time average over the period of the Larmor precession [42]:

$$
\mathrm{Q}_{\alpha \beta}^{\mathrm{L}}(\mathbf{r})=\frac{3 \hat{r}_{z}^{2}-1}{r^{3}} \frac{3 \delta_{z \alpha} \delta_{z \beta}-\delta_{\alpha \beta}}{2}
$$

Also in this case we truncate the dipolar interaction at a radius $R$ for computational purposes. Its Fourier transform then becomes (see Appendix A)

$$
\begin{array}{r}
\tilde{\mathrm{Q}}_{\alpha \beta}^{\mathrm{L}, r<R}(\mathbf{k})=-2 \pi\left(\hat{k}_{z}^{2}-1\right)\left(3 \delta_{z \alpha} \delta_{z \beta}-\delta_{\alpha \beta}\right) \times \\
{\left[\frac{1}{3}+\frac{k R \cos (k R)-\sin (k R)}{(k R)^{3}}\right] .}
\end{array}
$$

\section{B. Spin-3}

The spin- 1 condensate provides a useful system where the physical principles underlying the dipolar effects in a spinor BEC can be illustrated. Dipolar spin-1 BECs could potentially be realized using $\mathrm{Na}$ or $\mathrm{Rb}$ atoms by suppressing the $s$-wave scattering lengths via optical or microwave Feshbach resonances. However, large magnetic dipole moments are exhibited, e.g., by ${ }^{52} \mathrm{Cr}$, which is a spin-3 atom. In this case, the condensate 
wave function becomes a seven-component spinor with $\zeta=\left(\zeta_{+3}, \ldots, \zeta_{-3}\right)^{T}$ and a Hamiltonian density given by $[20,46,60]$

$$
\begin{aligned}
\mathcal{H}= & h_{0}+\frac{c_{0}}{2} n^{2}+\frac{c_{2}}{2} n^{2}|\langle\hat{\mathbf{F}}\rangle|^{2}+\frac{c_{4}}{2} n^{2}\left|A_{00}\right|^{2} \\
& +\frac{c_{6}}{2} n^{2} \sum_{j=-2}^{+2}\left|A_{2 j}\right|^{2}+\frac{c_{\mathrm{d}}}{2} \int D\left(\mathbf{r}, \mathbf{r}^{\prime}\right) d^{3} r^{\prime}
\end{aligned}
$$

where two additional interaction terms, compared with Eq. (21), appear as a result of the $s$-wave scattering of spin-3 atoms. These depend on the amplitudes

$$
A_{00}=\frac{1}{\sqrt{7}}\left(2 \zeta_{+3} \zeta_{-3}-2 \zeta_{+2} \zeta_{-2}+2 \zeta_{+1} \zeta_{-1}-\zeta_{0}^{2}\right)
$$

and

$$
\begin{aligned}
& A_{20}=\frac{1}{\sqrt{7}}\left(\frac{5}{\sqrt{3}} \zeta_{+3} \zeta_{-3}-\sqrt{3} \zeta_{+1} \zeta_{-1}+\sqrt{\frac{2}{3}} \zeta_{0}^{2}\right), \\
& A_{2 \pm 1}=\frac{1}{\sqrt{7}}\left(\frac{5}{\sqrt{3}} \zeta_{ \pm 3} \zeta_{\mp 2}-\sqrt{5} \zeta_{ \pm 2} \zeta_{\mp 1}+\sqrt{\frac{2}{3}} \zeta_{ \pm 1} \zeta_{0}\right), \\
& A_{2 \pm 2}=\frac{1}{\sqrt{7}}\left(\frac{10}{\sqrt{3}} \zeta_{ \pm 3} \zeta_{\mp 1}-\sqrt{\frac{20}{3}} \zeta_{ \pm 2} \zeta_{0}+\sqrt{2} \zeta_{ \pm 1}^{2}\right)
\end{aligned}
$$

respectively. The interaction strengths $c_{0,2,4,6}$ are found from the scattering lengths of the four spin channels of colliding spin- 3 atoms as $c_{0}=4 \pi \hbar^{2}\left(9 a_{4}+2 a_{6}\right) /(11 M)+$ $c_{0}^{\mathrm{d}}, c_{2}=4 \pi \hbar^{2}\left(a_{6}-a_{4}\right) /(11 M)+c_{2}^{\mathrm{d}}, c_{4}=4 \pi \hbar^{2}\left(11 a_{0}-\right.$ $\left.21 a_{4}+10 a_{6}\right) /(11 M)+c_{4}^{\mathrm{d}}$, and $c_{6}=4 \pi \hbar^{2}\left(11 a_{2}-18 a_{4}+\right.$ $\left.7 a_{6}\right) /(11 M)+c_{6}^{\mathrm{d}}$, where the coupling constants may again be modified by contact part of the DI. The dipolar interaction is again given by Eq. (3), where, the spin operator $\hat{\mathbf{F}}$ is now the vector of $7 \times 7$ spin- 3 Pauli matrices. From Eq. (15) seven coupled GPEs for the components of the spinor wave function may be derived. Using the spin-3 $\hat{\mathbf{F}}$ operator in Eqs. (3) and (6) yields the DI contribution, which can then be treated analogously to the spin-1 case.

The spin-3 BEC exhibits a complex family of phases exhibiting different symmetries [61]. Here we concentrate on ${ }^{52} \mathrm{Cr}$, where current measurements of the scattering lengths 62 64] predict an $A$-phase ground state with $|\langle\hat{\mathbf{F}}\rangle|=0$ in a uniform system. Nevertheless, the DI may influence the structure of singular vortices as they develop superfluid cores with nonzero spin.

\section{RESULTS}

We now employ the mean-field theory outlined in Section ஹto study the internal core structure of vortices. We find the vortex solutions by solving the coupled GPEs derived from Eq. 2 in the frame rotating with frequency $\Omega$ around the $z$ axis: $\mathcal{H} \rightarrow \mathcal{H}-\Omega\left\langle\hat{L}_{z}\right\rangle$, where $\hat{L}_{z}$ is the $z$ component of the angular-momentum operator. This is done using a successive overrelaxation method [65] to find stationary solutions in the spin- 1 model, while we have used imaginary-time propagation for particular results and to solve the spin-3 GPEs for ${ }^{52} \mathrm{Cr}$.

We first find our main results considering a spin-1 BEC. While higher-spin atoms are necessary to reach large magnetic dipole moment, the spin-1 system provides a useful model where the physics arising from the DI can be illustrated and compared with known results in a non-dipolar condensate. Since the DI is given by Eq. (3) regardless of the atomic spin and its effects arise from generic properties of the interaction and the spinor condensates, the physical principles illustrated by the results can be expected to apply more broadly also in higher-spin systems. Dipolar BECs can also be realized with weak dipolar interactions, provided that the other nonlinearities are even weaker (see aslo Appendix (B). We keep the spin-independent interaction strength fixed at $N c_{0}=10^{4} \hbar \omega \ell^{3}$, where $\ell$ is the oscillator length $\ell=\sqrt{\hbar /(M \omega)}$ of the harmonic trap and $N$ is the number of atoms in the condensate. We allow $c_{2}$ to vary around $c_{0} / c_{2} \simeq-216$, which corresponds to ${ }^{87} \mathrm{Rb}$, the most commonly used atom with FM interactions in spin1 experiments. We then study how the vortex structure varies with $c_{\mathrm{d}}$.

We further briefly consider a polar BEC with $c_{0} / c_{2} \simeq$ 28 , corresponding to ${ }^{23} \mathrm{Na}$. We then find the stable core structure of singular half-quantum vortices in a spin-3 ${ }^{52} \mathrm{Cr}$ BEC with with and without the corresponding DI, and analyze these in light of the spin-1 model.

\section{A. Weak magnetic field}

\section{Spin vortex}

For our spin- 1 model, we first consider a condensate in the FM interaction regime, $c_{2}<0$, such that in a uniform system $|\langle\hat{\mathbf{F}}\rangle|$ is maximized. In this case, different FM spinors are related by three-dimensional rotations of the orthonormal triad formed by $\langle\hat{\mathbf{F}}\rangle$ and two vectors perpendicular to it. The order-parameter space is therefore $\mathrm{SO}(3)$, which supports only two topologically distinct classes of vortices [20]: nonsingular coreless vortices and singly quantized singular vortices. Here we first consider the singular vortex, whose core structure in the absence of DI we studied in detail in Refs. 27, 31].

In a nondipolar condensate, the stabilization of a vortex line usually requires a sufficiently rapid external rotation. While the ground state in a rotating FM spin-1 condensate is generally predicted to be made up of coreless vortices [23, 24] , a singular FM vortex can also be energetically (meta-)stable for a range of trap-rotation frequencies [27], and is predicted to form the ground state when the coreless vortex is destabilized by conservation of a weak magnetization [30, 31]. Even though spin vortices that carry no mass circulation can form in a spinor BEC, one would not generally expect them to be ener- 


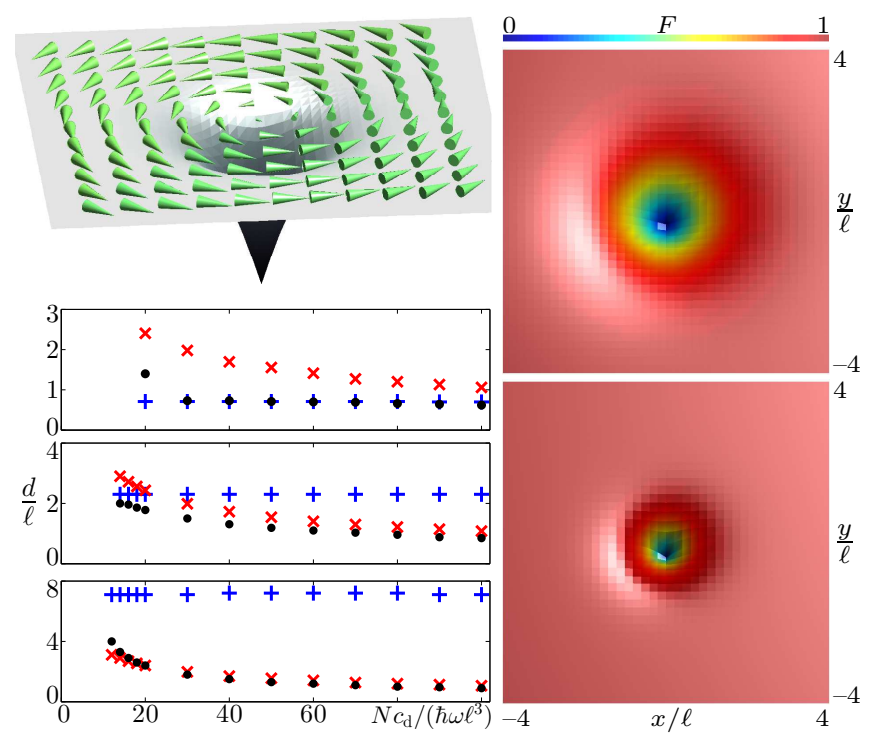

FIG. 1. Top left: Spin structure of a spin vortex in a nonrotating condensate. Surface plot indicates $|\langle\hat{\mathbf{F}}\rangle|$, showing the vortex core. Cones indicate $\langle\hat{\mathbf{F}}\rangle$, exhibiting a tangential disgyration that minimizes the DI energy. Bottom left: Size of the vortex core $(\bullet)$ compared with $\xi_{F}(+)$ and $\xi_{\mathrm{d}}^{\prime}(\times)$ as functions of $c_{\mathrm{d}}$ (see also Appendix (B). Subpanels from top to bottom: $N c_{2}=-463 \hbar \omega \ell^{3},-46.3 \hbar \omega \ell^{3}$, and $-4.63 \hbar \omega \ell^{3}$ $\left(N c_{0}=10^{4} \hbar \omega \ell^{3}\right)$. The spin vortex is stable above a critical $c_{\mathrm{d}}$ that depends on $c_{2}$. The core size is then well predicted by the smaller of the two healing lengths. Right: Surface plot of $F=|\langle\hat{\mathbf{F}}\rangle|$ [color (gray) scale] showing the different sizes of the vortex core for $N c_{\mathrm{d}}=16 \hbar \omega \ell^{3}$ (top) and $N c_{\mathrm{d}}=100 \hbar \omega \ell^{3}$ (bottom) for $N c_{2}=-46.3 \hbar \omega \ell^{3}$.

getically stable. In a FM spin-1 BEC, a spin vortex can be stabilized by magnetic fields, e.g., in a Ioffe-Pritchard trap [66], and a BEC with FM interactions initially in a polar state can be dynamically unstable towards spinvortex formation [25]. In the FM phase, mass circulation alone is not quantized and a spin vortex can continuously pick up angular momentum through local spin rotations to stabilize it in a rotating trap [22, 27].

When a magnetic DI is present, however, the situation changes due to the anisotropy of the interaction, which strives to arrange the dipoles in a head-to-tail configuration that minimizes the interaction energy. Beyond a critical $c_{\mathrm{d}}$, it then becomes possible for a singular spin vortex carrying no circulation to form in the ground state even in a nonrotating condensate $50-52]$. The structure of the stable spin vortex is shown in Fig. 1.

The tangential disgyration exhibited by the condensate spin is a consequence of the DI. The singular FM vortex in the spin-1 BEC can exhibit a wide range of associated spin textures that can be transformed into each other through local and continuous transformations. In addition to the tangential disgyration shown in Fig. 1, radial and cross disgyrations are possible, as well as asymptotically uniform spin textures. In the absence of DI, these different spin structures are energetically (near) degen- erate [27]. Here, this degeneracy is broken by the directional dependence of the DI. The tangential disgyration corresponds to the greatest head-to-tail alignment of the spins, and therefore minimizes the DI energy, energetically locking in the spin texture. The vortex can then be described by the spinor wave function

$$
\zeta=\frac{i}{\sqrt{2}}\left(\begin{array}{c}
-\sqrt{2} e^{-i \phi} \cos ^{2} \frac{\beta}{2} \\
\sin \beta \\
\sqrt{2} e^{i \phi} \sin ^{2} \frac{\beta}{2}
\end{array}\right),
$$

where $\phi$ is the azimuthal angel and $F_{z}=\cos \beta$. For $\beta=\pi / 2$, such that $\langle\hat{\mathbf{F}}\rangle$ lies in the $x y$ plane as in Fig. 1, the vortex is a pure spin vortex that carries no angular momentum.

In a scalar BEC, the superfluid density vanishes on the line singularity of the order parameter that constitutes a vortex line. In the multicomponent order parameter of a spinor BEC, by contrast, a vortex-line singularity can also be accommodated by exciting the wave function out of its ground-state manifold. In a spin1 BEC, the resulting filled vortex core becomes energetically favorable when $c_{2}$ is small compared with $c_{0}$, which is usually the case. This can be understood from the healing lengths arising from the contact-interaction terms. These are the density and spin healing lengths $\xi_{n}=\hbar /\left(2 M c_{0} n\right)^{1 / 2}$ and $\xi_{F}=\hbar /\left(2 M\left|c_{2}\right| n\right)^{1 / 2}$ that describe the characteristic length scales of deviations from the corresponding ground-state condition. By breaking the spin condition instead of depleting the density, the defect core can expand to the larger healing length and lower its energy [27, 56]. A singular FM vortex then develops a superfluid core exhibiting the polar phase on the line singularity. When the vortex is represented by Eq. (18), this corresponds to the $\zeta_{0}$ component occupying the singular lines in $\zeta_{ \pm}$.

In the dipolar spinor BEC considered here, an additional interaction term appears in the Hamiltonian density, Eq. (2). Unlike the interaction terms arising from the $s$-wave scattering, the DI term is nonlocal. However, it is still possible to associate with it a length scale

$$
\xi_{\mathrm{d}}=\frac{\hbar}{\sqrt{2 M\left|c_{\mathrm{d}}\right| n}}
$$

It was shown in Ref. [51] that dipole-induced spin textures such as the ground-state spin vortex can form when the extent of the condensate exceeds $\xi_{\mathrm{d}}$. Here we show that the dipolar healing length also interacts nontrivially with the other characteristic length scales to affect the vortex-core structure in the dipolar spinor condensate.

Specifically, we find that the dipolar healing length becomes important for the structure of a singular-vortex core when it is shorter than the spin healing length: $\xi_{\mathrm{d}} \lesssim \xi_{F}$. This is contrary to the nondipolar case, where the core structure of a singular vortex is determined by the largest healing length [27]. The healing lengths in that case are associated with different and independent ground state conditions (superfluid density and spin magnitude). In the case of magnetic DI, however, $\xi_{F}$ and $\xi_{\mathrm{d}}$ 
both relate to the condensate spin. In particular, when $c_{2}<0$ the contact interaction and the DI both energetically favor $|\langle\hat{\mathbf{F}}\rangle|=1$. Any perturbation of $|\langle\hat{\mathbf{F}}\rangle|$ must then heal back to the bulk value over the shortest of the spin-dependent healing lengths. Consequently, the size of the core becomes dependent on $c_{\mathrm{d}}$ when $\xi_{\mathrm{d}}$ becomes comparable to $\xi_{F}$ as illustrated in Fig. 1.

Unlike the contact interaction, the effective strength of the DI depends on the relative orientation of the dipoles. In a head-to-tail arrangement, the effective strength of the interaction is $-2 c_{\mathrm{d}}$ [cf. Eq. (3) for $\left.\mathbf{F}\left(\mathbf{r}^{\prime}\right)=\mathbf{F}(\mathbf{r})\right]$. In the context of the spin vortex shown in Fig. 1, $\xi_{F}$ should therefore be compared with $\xi_{\mathrm{d}}^{\prime} \equiv \xi_{\mathrm{d}} / \sqrt{2}$. In the bottom left panels of Fig. 1, we plot both $\xi_{F}$ and $\xi_{\mathrm{d}}^{\prime}$, together with the vortex core size (defined as the diameter of the core at $\left.|\langle\hat{\mathbf{F}}\rangle|=1-e^{-1}\right)$, as functions of $c_{\mathrm{d}}$. [For simplicity we here treat $c_{\mathrm{d}}$ as a free parameter within the spin-1 model system. Appendix B outlines how the dimensionless nonlinearity $N c_{\mathrm{d}} /\left(\hbar \omega \ell^{3}\right)$ can be varied also for fixed $c_{\mathrm{d}}$, corresponding to some particular magnetic dipole moment.] The middle subpanel corresponds to $N c_{2}=-46.3 \hbar \omega \ell^{3}$ (corresponding to ${ }^{87} \mathrm{Rb}$, whose physical dipole moment also gives $N c_{\mathrm{d}} \simeq 4.2 \hbar \omega \ell^{3}$, for comparison), while in the top and bottom panels, $c_{2}$ is one order of magnitude stronger and weaker, respectively. For the strong $c_{2}, \xi_{F}<\xi_{\mathrm{d}}^{\prime}$ over the range of the plot, and the core size remains nearly unaffected by $c_{\mathrm{d}}$ and is well predicted by $\xi_{F}$ (except at the very onset of stability). Conversely, for the weak $c_{2}, \xi_{F}>\xi_{\mathrm{d}}^{\prime}$, with the latter quantity corresponding well to the core size. In the middle panel, $\xi_{F} \simeq \xi_{\mathrm{d}}^{\prime}$ and the two cross as $c_{\mathrm{d}}$ increases. For large $c_{\mathrm{d}}$ the core size follows $\xi_{\mathrm{d}}^{\prime}$, while at small $c_{\mathrm{d}}$ the influence of the now smaller $\xi_{F}$ becomes evident. These fairly simple principles then characterize the behavior of a singulardefect core as DI is varied.

For a nonrotating cloud in a 3D isotropic trap, there is no preferred direction for the spin-vortex line (in the absence of Zeeman shifts). In Fig. [1 the vortex line coincides with the $z$ axis, while the left panel of Fig. 2 shows a spin vortex line in the $x y$ plane. Considering now a slowly rotating trap, the axis of rotation represents a preferred spatial direction. Vortices stabilized by rotation would then form parallel to the rotation axis, as in the nondipolar case [27]. In a highly oblate dipolar spinor condensate, the spin vortex with axial symmetry around the $z$ direction persists up to a critical rotation frequency [53]. For our isotropic trap, however, we find that the ground state in a slowly rotating condensate exhibits a spin vortex forming perpendicularly to the rotation axis when $c_{\mathrm{d}}$ is sufficiently large (Fig. (2). While a solution with a spin vortex parallel to the rotation also exists, this has a higher energy. The orientation of the vortex line shows that similarly to the nonrotating case, the vortex forms due to minimization of the DI energy, rather than because of the rotation. The effect of the rotation is instead to increasingly bend the vortex line, as illustrated in Fig. 2 .

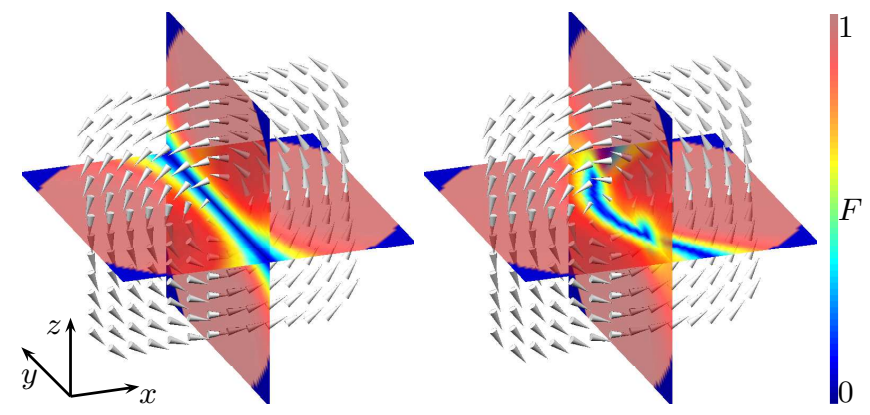

FIG. 2. Spin vortex in nonrotating (left) and slowly rotating, $\Omega=0.10 \omega$ (right), trap. Color map (gray scale) shows $F=$ $|\langle\hat{\mathbf{F}}\rangle|$ highlighting the polar vortex core, while cones show the spin vector in the $y=0$ plane. In the rotating system, the vortex line forms perpendicular to the rotation axis. $N c_{0}=$ $10^{4} \hbar \omega \ell^{3}, N c_{2}=-46.3 \hbar \omega \ell^{3}, N c_{\mathrm{d}}=50 \hbar \omega \ell^{3}$.

\section{Coreless vortex}

When the condensate rotates sufficiently rapidly, we find that the ground state is a coreless vortex along the rotation axis. This was also found to be the case in the highly oblate trap in Ref. [53]. Coreless vortices are also predicted to make up the ground state in a rotating nondipolar FM condensate [23, 24], unless destabilized through conservation of a sufficiently weak magnetization [30]. The coreless vortex is characterized by a nonsingular spin texture in which the superfluid circulation varies continuously as the spin bends from $\langle\hat{\mathbf{F}}\rangle=\hat{\mathbf{z}}$ on the vortex line toward its asymptotic direction. The boundary condition on the spin texture away from the vortex line is however not fixed, allowing it to adapt to the imposed rotation, bending more sharply towards the $-\hat{\mathbf{z}}$ direction as rotation increases.

The DI introduces a competing mechanism that strives to align the spins in the head-to-tail configuration of a tangential disgyration similar to the spin vortex. This leads to the formation of a coreless vortex with the spin texture shown in Fig. 3, where the spin vector bends gradually into the tangential disgyration. Far from the vortex line, the DI thus determines the spin texture by the same mechanism as for the spin vortex. Note, however, that while the two vortices appear superficially similar, both exhibiting tangential disgyrations of the spin vector at large length scales, their topology is distinctly different. This is easily established from the complex phases of the individual spinor components, which exhibit $(2 \pi, 0,-2 \pi)$ winding in the singular spin vortex, but wind by $(0,2 \pi, 4 \pi)$ in the coreless vortex. In the latter case, the vortex can be removed through purely local spin rotations, provided that the value of the spin is free to rotate at the edge of the cloud.

The structure of the coreless vortex can be understood as the result of competition between rotation and DI. While the spin texture strives to adapt to the imposed rotation asymptotically forming an angle with the $z$ axis 


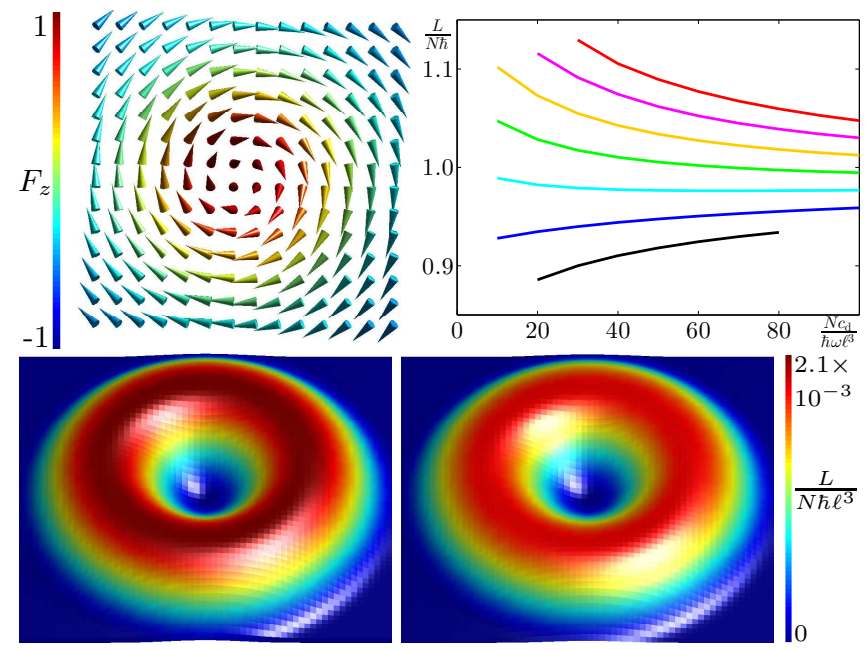

FIG. 3. Top left: Spin texture of the coreless vortex for $\Omega=0.175 \omega$ and $N c_{\mathrm{d}}=10 \hbar \omega \ell^{3}$. Cones show the spin vector in the $x, y$ plane, perpendicular to the vortex line, with the color (gray) scale indicating the $z$ component. The DI causes the condensate spin to bend toward a tangential disgyration away from the vortex line. Top right: Total angular momentum carried by the coreless vortex as a function of $c_{\mathrm{d}}$. The lines from bottom to top correspond to trap rotation $\Omega=0.14 \omega$ through $\Omega=0.20 \omega$ in steps of $0.01 \omega$. The varying dependence on $c_{\mathrm{d}}$ and the convergent behavior at large values is the result of the spin texture simultaneously adapting to DI and imposed rotation. Bottom: Angular-momentum density distribution for $\Omega=0.18 \omega$ and $N c_{\mathrm{d}}=10 \hbar \omega \ell^{3}$ (left) and $100 \hbar \omega \ell^{3}$ (right). The panels show the same $13 \ell$ by $13 \ell$ cut-out and use the same color (gray) scale. In all panels $N c_{0}=10^{4} \hbar \omega \ell^{3}, N c_{2}=-46.3 \hbar \omega \ell^{3}$

that depends on the rotation frequency, the DI strives to align the asymptotic texture in the $x y$ plane. This competition is reflected in the total angular momentum $L$ carried by the vortex, which becomes dependent on $c_{\mathrm{d}}$ at fixed trap rotation. When the trap rotates slowly, the rotation alone is not enough to bring the asymptotic texture into the $x y$ plane. Increasing $c_{\mathrm{d}}$ will then result in a more sharply bending texture that carries additional angular momentum, such that $L$ increases as a function of $c_{\mathrm{d}}$. On the other hand, a rapid rotation causes the asymptotic spin texture to acquire a negative $F_{z}$ component in order to provide sufficient circulation. Increasing $c_{\mathrm{d}}$ then has the opposite effect, causing the spin to bend less sharply in order to bring it more in line with the $x y$ plane. This causes $L$ to decrease with with DI strength. Consequently, as the DI becomes more dominant with increasing $c_{\mathrm{d}}, L$ becomes less sensitive to the trap rotation frequency.

\section{Polar condensate}

The DI couples to the condensate spin through Eq. (3). So far we have explored how this leads to consequences for the formation, stability and structure of in the FM phase, where $|\langle\hat{\mathbf{F}}\rangle|$ is maximized in the bulk condensate. In the polar phase of the spin-1 BEC, $|\langle\hat{\mathbf{F}}\rangle|=0$ in a uniform system. However, when a singular vortex is present, nonzero $|\langle\hat{\mathbf{F}}\rangle|$ can appear in the defect core, and DI can still affect its structure. The physics, however, exhibits differences from the FM case, where the contact and dipolar interactions both strive to maximize the condensate spin. Here, by contrast, the interactions compete, with the contact interaction favoring $|\langle\hat{\mathbf{F}}\rangle|=0$. The size of a superfluid core with nonzero spin is then not limited by the dipolar interaction, which now favors breaking of the ground-state spin condition. One may then expect the presence of the DI to lead to an enlarged core, and our numerical simulations confirm these simple principles.

We explore this by considering a stable singular halfquantum vortex in a rotating system. We keep $c_{0}$ the same as in the FM examples, but now take $c_{0} / c_{2} \simeq 28$ corresponding to ${ }^{23} \mathrm{Na}$. Energy relaxation causes the vortex to develop a superfluid core that breaks the groundstate spin condition, reaching the FM phase on the singular line [31]. In the absence of DI the size of the vortex core is determined by the spin healing length $\xi_{F}$. We find that as the strength of the DI increases from $c_{\mathrm{d}}=0$ to $c_{0} / c_{\mathrm{d}}=200$, the size of the vortex core increases by $\sim 35 \%$ for $N c_{0}=10^{4} \hbar \omega \ell^{3}$ and rotation frequency in the range $0.12 \omega \leq \Omega \leq 0.17 \omega$.

\section{B. Precession-averaged dipolar interaction in a magnetic field}

In experiment, the condensate may be subject to residual or deliberately imposed external magnetic fields. In the presence of the magnetic field, the condensate spin exhibits precession around the field direction at the Larmor frequency $\omega_{\mathrm{L}}$. If the field is sufficiently strong, the Larmor precession will be rapid compared with the spin dynamics resulting from the DI. On the latter time scale, the condensate then experiences an effective DI that corresponds to the averaging of the bare DI over the period of the Larmor precession. The resulting reduced DI is given by Eq. (13). This removes some of the anisotropy of the bare DI and therefore leads to degeneracy between some spin configurations that would otherwise have different energies. Here we show that this can have a profound effect on the spin structure of singular vortices. Figure 4 shows the vortex core and spin texture of a stable singular vortex in a rotating system. For sufficiently small $c_{\mathrm{d}}$, the vortex is axially symmetric, exhibiting a radial disgyration in the $x y$ components of the spin vector.

Increasing $c_{\mathrm{d}}$ results in a deformation of the vortex core, breaking the axial symmetry. The spins rotate toward a configuration where the asymptotic spin texture exhibits a nearly uniform projection onto the $x y$ plane, while $F_{z}$ bends across the condensate. At the same time, the polar core of the vortex deforms to exhibit an ellip- 


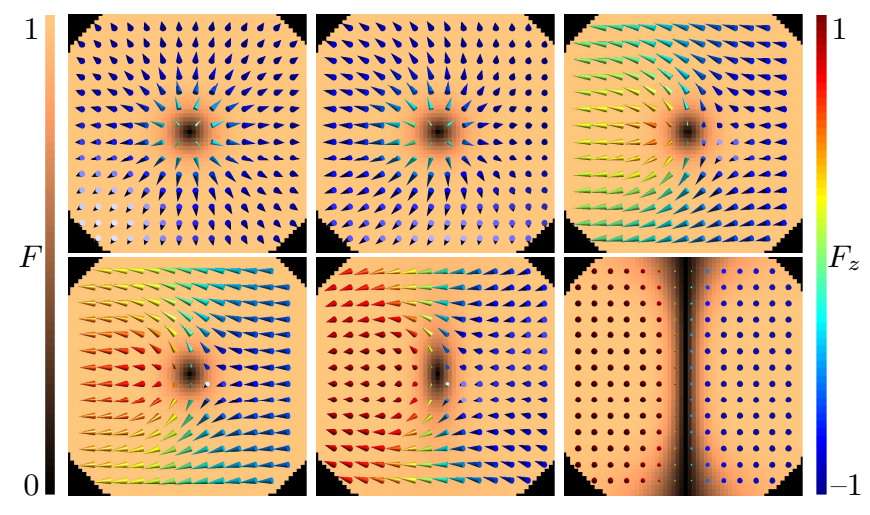

FIG. 4. Structure of the stable singular-vortex core $(z=0$ cross section, perpendicular to the vortex line) as the effective DI averaged over the Larmor precession increases. Color map (gray scale) shows $F=|\langle\hat{\mathbf{F}}\rangle|$, while cones show the spin vector $\langle\hat{\mathbf{F}}\rangle$ the color (shade) of the cones indicates $\left.F_{z}\right]$. From top left to bottom right $N c_{\mathrm{d}}=0,6,8,10,50$ and $100 \hbar \omega \ell^{3}$. The core deforms by breaking axial symmetry to form an ellipsoidal cross section. Eventually the core covers the diameter of the condensate and forms a domain wall between regions of $F_{z}=$ \pm 1 . In all panels $N c_{0}=10^{4} \hbar \omega \ell^{3}, N c_{2}=-46.3 \hbar \omega \ell^{3}$, and $\Omega=0.13 \omega$.

tical cross section whose eccentricity increases with $c_{\mathrm{d}}$. Eventually the deformation of the vortex core becomes large enough that its extent along the major axis of the ellipse reaches the condensate size. As shown in Fig. 4 the condensate the exhibits a polar domain wall separating two halves of the cloud with oppositely aligned spin $\langle\hat{\mathbf{F}}\rangle= \pm \hat{\mathbf{z}}$. In the isotropic spinor condensate, there is no analog of this deformation when the DI cannot be averaged over the Larmor precession period. However, a similar anisotropic deformation has been predicted in a twodimensional, scalar BEC with fixed dipole moments 67]

\section{Singular vortex in a spin-3 ${ }^{52} \mathrm{Cr}$ BEC}

The spin-1 BEC provides a good model system for theoretically exploring the physical principles of DI effects on vortices in spinor BECs. However, a stronger dipole moment of $6 \mu_{\mathrm{B}}$ is found in ${ }^{52} \mathrm{Cr}$, which can be used to create a spin-3 condensate [46, 60]. Measurements of the $s$-wave scattering lengths $\left[62\left[64\right.\right.$ yield $c_{0} / c_{2} \simeq 20$, $c_{0} / c_{4} \simeq-4.6, c_{0} / c_{6} \simeq-1.5$ for the contact-interaction strengths in Eq. (15), while $c_{0} / c_{\mathrm{d}} \simeq 177$. The groundstate determined by the $s$-wave interaction is then the socalled $A$-phase in a uniform system [61], with a representative order-parameter $\zeta=(1 / \sqrt{2}, 0,0,0,0,0,1 / \sqrt{2})^{T}$. This exhibits $|\langle\hat{\mathbf{F}}\rangle|=0$ and the order parameter has the discrete hexagonal symmetry of the dihedral-6 group $D_{6}$ 61, 68, 69], illustrated in Fig. 5] using the spherical- harmonics representation

$$
Z(\theta, \phi)=\sum_{m=-3}^{+3} Y_{3, m}(\theta, \phi) \zeta_{m}
$$

In a biaxial-nematic spin-2 BEC, the related but simpler dihedral-4 point-group symmetry already leads to highly complex core structures of a half-quantum vortex [32]. As a result of the $D_{6}$ symmetry, the spin- $3 A$-phase vortices are also non-Abelian (i.e., the different topological charges do not all commute), leading to the restricted reconnection dynamics of vortices also predicted in the cyclic and biaxial-nematic spin-2 phases [32, 70].

The $A$-phase $D_{6}$ order parameter supports a halfquantum vortex where the $\pi$ winding of the condensate phase is compensated by a $\pi / 3$ spin rotation. This is the simplest vortex that carries angular momentum and could therefore be stabilized by rotation. Figure 5 shows the relaxed core structure of one out of a pair of such half-quantum vortices in a condensate with and without the DI corresponding to ${ }^{52} \mathrm{Cr}$, for the case where any external magnetic field is negligible (i.e., the spin precession is not assumed to be rapid). We find that energy relaxation leads to the condensate approaching the $H$-phase [61] on the singular line. A representative $H$-phase order parameter can be written $\zeta=(\sqrt{(2+F) / 5}, 0,0,0,0, \sqrt{(3-F) / 5}, 0)^{T}$, where $F=|\langle\hat{\mathbf{F}}\rangle|$. This phase exhibits a five-fold rotational symmetry, shown in Fig. 5. The bottom left panel also uses the spherical-harmonics representation, Eq. (20), to illustrate the change of the order parameter form the bulk $A$-phase to the vortex core. The $H$-phase further exhibits a parameter-dependent condensate spin magnitude that is determined by energy relaxation. Here we find $|\langle\hat{\mathbf{F}}\rangle|>0$ in the vortex core, which therefore breaks the ground-state spin condition. The effects of DI are then similar to the polar half-quantum vortex with FM core in the spin-1 model (section III A 3), where increasing DI leads to an increase in core size since the DI favors the nonzero spin. In the spin-3 vortex, however, $|\langle\hat{\mathbf{F}}\rangle|$ is not restricted to a particular value on the vortex line, but is determined by energy relaxation and depends on the interaction parameters. Comparing the stable vortex cores, we find a slightly increased spin magnitude in the presence of DI, illustrating the general principle that was also demonstrated for the spin-1 dipolar BEC.

However, the presence of the DI is also reflected in the internal spin texture of the vortex core, which is reminiscent of the coreless vortex in Fig. 3. By the same mechanism, the anisotropy of the DI here leads to the formation of a spin texture across the vortex core that approaches a tangential disgyration as shown in Fig. 5 , In a condensate where $\xi_{F}$, and therefore the vortex core, is not small on the scale of experimental resolution, this effect could be observed in experiment. 

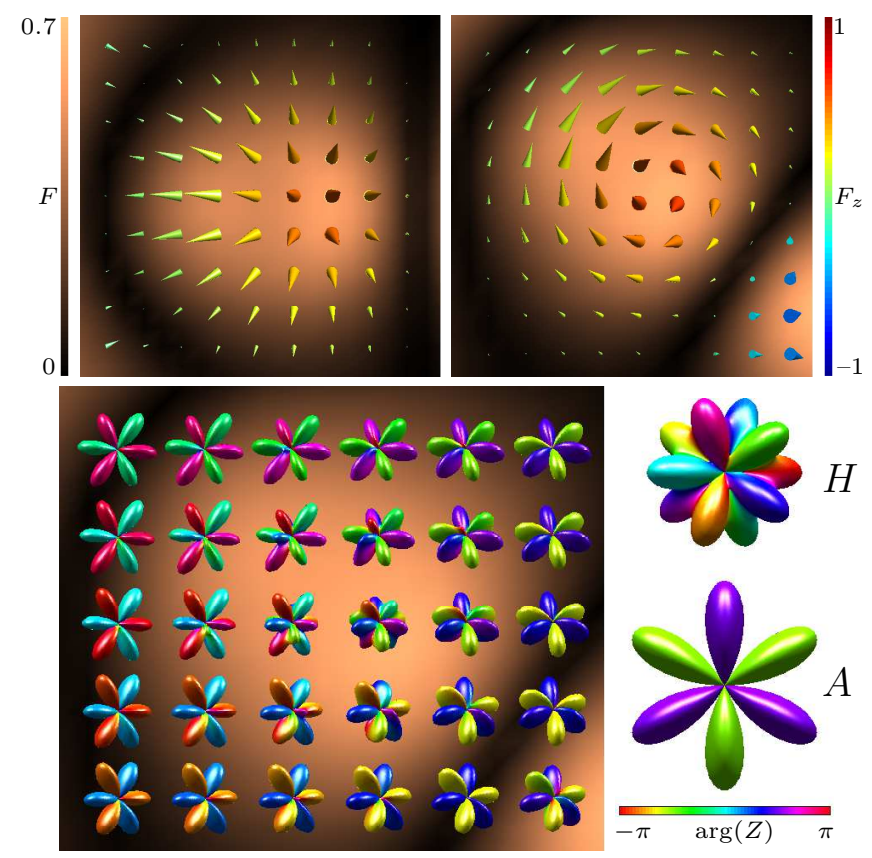

FIG. 5. Top: Core structure of a singular half-quantum vortex in the $A$-phase of a spin-3 BEC in the absence (left) and presence (right) of DI corresponding to ${ }^{52} \mathrm{Cr}$. Color map (gray scale) shows $F=|\langle\hat{\mathbf{F}}\rangle|$, while cones show the spin vector $\langle\hat{\mathbf{F}}\rangle$ [the color (shade) of the cones indicates $F_{z}$ ). The panels show a $2.4 \ell \times 2.4 \ell$ region around the vortex line, which is stable together with a second half-quantum vortex (not shown) in a system rotating with frequency $\Omega=0.43 \omega$. The contact interaction corresponds to ${ }^{52} \mathrm{Cr}$ with $N c_{0}=10^{3} \hbar \omega \ell^{3}$. Bottom left: Change of the order parameter symmetry showing the transition from the $A$-phase bulk towards the $H$-phase in the core when DI is present [background color map (gray scale) shows $F$ for reference]. Bottom right: Spherical-harmonics representations of the $A$ - and $H$-phase order parameters for reference.

\section{CONCLUSIONS}

We have demonstrated how DI can have several pronounced effects on the internal structure of vortices in spinor BECs, and determined and analyzed the stable core structure of singular vortices in a spin- $3{ }^{52} \mathrm{Cr}$ condensate. In addition to exhibiting relatively strong dipolar interactions, the ${ }^{52} \mathrm{Cr}$ interaction parameters predict a ground-state order parameter exhibiting a hexagonal point-group symmetry that makes it a candidate for experimental observation of non-Abelian vortices.

We used a spin-1 model system to analyze the underlying physical principles and established simple criteria that determine the defect core structure in the presence of DI. We have shown how a new characteristic length scale arising from the DI adds to the hierarchy of healing lengths to restrict the size of singular-vortex cores when $\mathrm{DI}$ and $s$-wave scattering both favor the ground-state spin condition (e.g., in the spin-1 FM phase), but can lead to core enlargement when they compete, as in the spin-
1 polar phase. These dipolar effects arise from generic properties of the interaction and the spinor system and our results can therefore be expected to apply generally across condensates of atoms with different atomic spin.

We have also shown how the spin ordering induced by the anisotropy of the DI has several different manifestations in both singular and nonsingular vortices. These include a nontrivial interaction dependence of the angular momentum carried by a coreless vortex, arising as a result of competition between dipolar spin ordering and rotation, as well as the deformation of a singular vortex when the DI is modified by a sufficiently strong magnetic field. The spin ordering can also give rise to internal spin textures in the superfluid vortex cores with nonzero spin, which are potentially observable in non-Abelian vortices in ${ }^{52} \mathrm{Cr}$ condensates. Similar studies of the effects of DI could be extended beyond vortices to other more complex defects and textures [56, 71, 72] in which case their symmetries and stability properties could be altered.

\section{ACKNOWLEDGMENTS}

We acknowledge financial support from the EPSRC. The numerical results were obtained using the Iridis 4 high-performance computing facility at the University of Southampton. We acknowledge discussions with T. P. Simula.

\section{Appendix A: Explicit derivation of Fourier Transforms}

In numerical computations, it is convenient to calculate DI contributions in Fourier space, where the convolution integrals arising from the long-range nature of the DI become a simple matter of multiplication of Fourier transforms. However, the $\delta$-function contribution to the magnetic dipole field and its absorption into the $s$-wave interaction introduces subtleties into the derivation of these Fourier transforms. Here we first carefully derive the Fourier transform of the magnetic dipole field, keeping track of all contributions, and show how it yields Eq. (11) after explicitly subtracting the contact-interaction part. We then show how the Fourier transform is modified by the introduction of a spherical long-range cut-off and also indicate how the corresponding derivation is modified when the DI is averaged over a rapid Larmor precession.

The magnetic field $\mathbf{B}(\mathbf{r})$ from a magnetic point dipole $\mathbf{m}$ at the origin is given by [73]

$$
\mathbf{B}(\mathbf{r})=\frac{\mu_{0}}{4 \pi r^{3}}[3 \hat{\mathbf{r}}(\mathbf{m} \cdot \hat{\mathbf{r}})-\mathbf{m}]+\frac{2 \mu_{0}}{3} \mathbf{m} \delta(r),
$$

where $\hat{\mathbf{r}}=\mathbf{r} / r$. We can write Eq. (A1) on tensor form as

$$
B_{\alpha}=\frac{\mu_{0}}{4 \pi} \sum_{\beta} \mathrm{B}_{\alpha \beta} m_{\beta},
$$


where

$$
\mathrm{B}_{\alpha \beta}(\mathbf{r})=\frac{3 \hat{r}_{\alpha} \hat{r}_{\beta}-\delta_{\alpha \beta}}{r^{3}}+\frac{8}{3} \delta_{\alpha \beta} \delta(r) .
$$

The $\delta$-function contribution to the field follows from

$$
\int_{\delta} \mathbf{B} d^{3} r=\frac{2 \mu_{0}}{3} \mathbf{m}
$$

with the convention that the integral of the first term in Eq. (A1) vanishes on any infinitesimal sphere $\delta$ surrounding the point dipole (integrating over angles first to make the integral converge). In writing the dipolar GrossPitaevskii Hamiltonian, Eq. (2), however, this contactinteraction contribution is absorbed by the $s$-wave interaction, yielding an effective field

$$
\mathbf{B}^{\prime}(\mathbf{r}) \equiv \mathbf{B}(\mathbf{r})-\frac{2 \mu_{0}}{3} \mathbf{m} \delta(\mathbf{r})
$$

that corresponds to the tensor

$$
\mathrm{Q}_{\alpha \beta} \equiv \mathrm{B}_{\alpha \beta}-\frac{8}{3} \delta_{\alpha \beta} \delta(r)=\frac{3 \hat{r}_{\alpha} \hat{r}_{\beta}-\delta_{\alpha \beta}}{r^{3}},
$$

appearing in Eqs. (8) and (9) and whose Fourier transform is needed in Eq. (10).

In finding the Fourier transforms of $B$ and $Q$ we need to ensure that the $\delta$-function contribution and its subtraction are correctly accounted for. In order to compute the Fourier transform, it is convenient to rewrite the dipole field as

$$
\mathbf{B}=\frac{\mu_{0}}{4 \pi}(\mathbf{m} \times \nabla) \times \nabla \frac{1}{r} .
$$

In the tensor notation, this corresponds to rewriting B as

$$
\mathrm{B}_{\alpha \beta}=\sum_{\gamma \mu \nu} \epsilon_{\alpha \gamma \mu} \epsilon_{\gamma \beta \nu} \partial_{\nu} \partial_{\mu} \frac{1}{r}
$$

where $\epsilon_{\alpha \beta \gamma}$ is the fully antisymmetric Levi-Civita tensor. It is straightforward to check that Eq. (A7) gives the correct field away from the origin [corresponding to the first term of Eq. (A1)]. However, we now need to check that the condition (A4) is satisfied. To do this, we first rewrite the integral of $\mathbf{B}$ as a surface integral by rearranging the cross products and using the divergence theorem:

$$
\begin{aligned}
I & \equiv \int_{\delta} \frac{\mu_{0}}{4 \pi}(\mathbf{m} \times \nabla) \times \nabla \frac{1}{r} d^{3} r \\
& =-\int_{\partial \delta} \frac{\mu_{0}}{4 \pi} \hat{\mathbf{r}} \times(\mathbf{m} \times \nabla) \frac{1}{r} d S .
\end{aligned}
$$

Then using $\boldsymbol{\nabla}(1 / r)=\mathbf{r} / r^{3}$ and writing $d S=$ $r^{2} d(\cos \theta) d \phi$ in spherical coordinates, the integral be- comes

$$
\begin{aligned}
I & =\frac{\mu_{0}}{4 \pi} \int_{\partial \delta} \hat{\mathbf{r}} \times(\mathbf{m} \times \hat{\mathbf{r}}) d(\cos \theta) d \phi \\
& =\frac{\mu_{0}}{4 \pi} \int_{\partial \delta}[-\hat{\mathbf{r}}(\mathbf{m} \cdot \hat{\mathbf{r}})+\mathbf{m}] d(\cos \theta) d \phi, \\
& =\frac{2 \mu_{0}}{3} \mathbf{m},
\end{aligned}
$$

in agreement with Eq. (A4). We have thus verified that Eqs. (A7) and (A8) correctly yield the magnetic dipole field, Eq. (A1), including the $\delta$-function contribution 74]

We can now proceed to find the Fourier transform of the full magnetic dipole field as

$$
\begin{aligned}
\tilde{\mathbf{B}}(\mathbf{k}) & =\int e^{-i \mathbf{k} \cdot \mathbf{r}} \frac{\mu_{0}}{4 \pi}(\mathbf{m} \times \nabla) \times \nabla \frac{1}{r} d^{3} r \\
& =-\frac{\mu_{0}}{k^{2}}(\mathbf{m} \times \mathbf{k}) \times \mathbf{k},
\end{aligned}
$$

where we have first used the vector identity

$$
\int_{V} f(\mathbf{v} \times \nabla) \times \nabla g d^{3} r=\int_{V} g(\mathbf{v} \times \nabla) \times \nabla f d^{3} r,
$$

and then find the remaining integral as

$$
\begin{aligned}
\int \frac{e^{-i \mathbf{k} \cdot \mathbf{r}-\mu r}}{r} d^{3} r & =\frac{2 \pi}{i k}\left(\frac{1}{-i k-\mu}-\frac{1}{i k-\mu}\right) \\
& \stackrel{\mu \rightarrow 0}{\longrightarrow} \frac{4 \pi}{k^{2}}
\end{aligned}
$$

using the convergence factor $\mu$, to yield the right-hand side of Eq. (A11). Rewriting Eq. (A11) using vector identities we arrive at

$$
\tilde{\mathbf{B}}(\mathbf{k})=-\mu_{0}[\hat{\mathbf{k}}(\mathbf{m} \cdot \hat{\mathbf{k}})-\mathbf{m}] .
$$

From the tensor notation $\tilde{B}_{\alpha}(\mathbf{k})=\left[\mu_{0} /(4 \pi)\right] \sum_{\beta} \tilde{\mathrm{B}}_{\alpha \beta} m_{\beta}$, it follows immediately that

$$
\tilde{\mathrm{B}}_{\alpha \beta}(\mathbf{k})=-4 \pi\left(\hat{k}_{\alpha} \hat{k}_{\beta}-\delta_{\alpha \beta}\right) .
$$

When the contact part of the interaction is absorbed by the $s$-wave interaction, however, we need to consider instead the Fourier transform of Eq. (A5), which is immediately found from linearity as

$$
\tilde{\mathbf{B}}^{\prime}(\mathbf{k})=-\frac{\mu_{0}}{3}[3 \hat{\mathbf{k}}(\mathbf{m} \cdot \hat{\mathbf{k}})-\mathbf{m}]
$$

Writing this in tensor notation such that $\tilde{B}_{\alpha}^{\prime}(\mathbf{k})=$ $\left[\mu_{0} /(4 \pi)\right] \sum_{\beta} \tilde{Q}_{\alpha \beta} m_{\beta}$ immediately yields Eq. (11).

It is common in the literature (see, e.g., Refs. [20, 58]) to arrive at Eq. (11), or its special case for aligned dipoles, by ignoring the $\delta$-function contribution in Eq. (A1), considering only $\mathbf{B}^{\prime}$ and expressing $\mathbf{Q}$ in terms of spherical harmonics as 


$$
\mathrm{Q}(\mathbf{r})=-\sqrt{\frac{6 \pi}{5}} \frac{1}{r^{3}}\left(\begin{array}{c}
\sqrt{\frac{2}{3}} Y_{2,0}(\hat{\mathbf{r}})-Y_{2,2}(\hat{\mathbf{r}})-Y_{2,-2}(\hat{\mathbf{r}}) \\
i Y_{2,2}(\hat{\mathbf{r}})-i Y_{2,-2}(\hat{\mathbf{r}}) \\
Y_{2,1}(\hat{\mathbf{r}})-Y_{2,-1}(\hat{\mathbf{r}})
\end{array}\right.
$$

One then makes use of the expansion of a plane wave in terms of spherical harmonics to find

$$
\int e^{-i \mathbf{k} \cdot \mathbf{r}} Y_{l, m}(\hat{\mathbf{r}}) d \Omega=4 \pi(-i)^{l} j_{l}(k r) Y_{l, m}(\hat{\mathbf{k}})
$$

where $j_{l}$ is the spherical Bessel function of order $l$. The radial integral

$$
\int_{0}^{\infty} \frac{j_{2}(k r)}{r^{3}} r^{2} d r=\int_{0}^{\infty} u^{2}\left(\frac{1}{u} \frac{d}{d u}\right)^{2} \frac{\sin u}{u} \frac{d u}{u}=\frac{1}{3}
$$

where $u=k r$, can then be combined with Eqs. (A17) and (A18) to find Eq. (11). Note, however, that this derivation drops the contact term from the outset and the Fourier integral is made to converge only by integrating over angles first in Eq. (A18). Nevertheless, having now established that the use of Eqs. A18) and (A19) does in fact give the correct result when the contact part of the DI is absorbed in the $s$-wave interaction, this provides a convenient way to include the long-range cut-off that is necessary in numerical computations, as this affects the Fourier integral only away from the origin. Then truncating the DI at a radius $R$, Eq. A19 becomes

$$
\int_{0}^{R} \frac{j_{2}(k r)}{r^{3}} r^{2} d r=\frac{1}{3}+\frac{k R \cos (k R)-\sin (k R)}{(k R)^{3}}
$$

from which Eq. (12) follows immediately. This is the immediate generalization of the spherical cut-off found by Ronen et al [58] for aligned dipoles in a scalar BEC to free dipoles.

Finally we consider the effective DI arising when the interaction is averaged over the Larmor precession period in the presence of a sufficiently strong magnetic field. We can rewrite the corresponding tensor $Q^{L}$ given by Eq. (13)

$$
\left.\begin{array}{cc}
i Y_{2,2}(\hat{\mathbf{r}})-i Y_{2,-2}(\hat{\mathbf{r}}) & Y_{2,1}(\hat{\mathbf{r}})-Y_{2,-1}(\hat{\mathbf{r}}) \\
\sqrt{\frac{2}{3}} Y_{2,0}(\hat{\mathbf{r}})+Y_{2,2}(\hat{\mathbf{r}})+Y_{2,-2}(\hat{\mathbf{r}}) & -i Y_{2,1}(\hat{\mathbf{r}})-i Y_{2,-1}(\hat{\mathbf{r}}) \\
-i Y_{2,1}(\hat{\mathbf{r}})-i Y_{2,-1}(\hat{\mathbf{r}}) & -2 \sqrt{\frac{2}{3}} Y_{2,0}(\hat{\mathbf{r}})
\end{array}\right) .
$$

as

$$
\mathrm{Q}_{\alpha \beta}^{\mathrm{L}}(\mathbf{r})=\frac{Y_{2,0}(\hat{\mathbf{r}})}{r^{3}} \frac{3 \delta_{z \alpha} \delta_{z \beta}-\delta_{\alpha \beta}}{2},
$$

and proceed as above. Then, from Eqs. (A18) and (A20), Eq. (14) follows immediately.

\section{Appendix B: Relative strength of DI}

For our studies, we have employed a suitably simple spin-1 model system to illustrate the physics arising from DI. The DI coupling constant $c_{\mathrm{d}}$ is then regarded as a freely variable parameter. For physical atoms, however, the atomic dipole moment, and therefore $c_{\mathrm{d}}$, is a fixed quantity. In this Appendix we briefly outline how the dipolar nonlinearity (as given in Fig. 1) can also be varied within the same spin-1 model by adjusting trap parameters and scattering lengths.

The effective nonlinearities in the GPEs, expressed in dimensionless units as in Fig. 11 scale with the number of atoms $N$ in the condensate and the trap frequency $\omega$ as $\sim N \omega^{1 / 2}$. Therfore the DI nonlinearity $N c_{\mathrm{d}} /\left(\hbar \omega \ell^{3}\right)$, used in the figure, can be varied also for constant $c_{\mathrm{d}}$ by adjusting $N$ and/or $\omega$. We can illustrate this principle using ${ }^{87} \mathrm{Rb}$ as a particular example: $N c_{0}=10^{4} \hbar \omega \ell^{3}$, in Fig. 11 then corresponds to $N \simeq 5 \times 10^{5}$ atoms in an $\omega \simeq 2 \pi \times 10 \mathrm{~Hz}$ trap, and the physical magnetic dipole moment gives $N c_{\mathrm{d}} \simeq 4.2 \hbar \omega \ell^{3}$. By doubling the atom number to $N=10^{6}$ and increasing the trap frequency to $\omega \simeq 2 \pi \times 60 \mathrm{~Hz}$, we can reach $N c_{\mathrm{d}} \simeq 20 \hbar \omega \ell^{3}$.

However, adjusting the trap parameters also scales the contact-interaction nonlinearity $N c_{0} /\left(\hbar \omega \ell^{3}\right)$. This can be prevented by simultaneously suppressing the contactinteraction coupling constant $c_{0}$. The suppression may be achieved using ac Stark shifts to access Feshbach resonances for the $s$-wave scattering lengths without freezing out the atomic spin [29, 55]. In the ${ }^{87} \mathrm{Rb}$ example, the required suppression is on the order of a factor $\sim 5$. Using these techniques, a strongly dipolar BEC could also be achieved using ${ }^{85} \mathrm{Rb}$ where scattering lengths are tunable across orders of magnitude [75].
[1] A. Griesmaier, J. Werner, S. Hensler, J. Stuhler, and T. Pfau, Phys. Rev. Lett. 94, 160401 (2005).

[2] B. Pasquiou, E. Maréchal, G. Bismut, P. Pedri, L. Vernac, O. Gorceix, and B. Laburthe-Tolra, Phys. Rev. Lett. 106, 255303 (2011).
[3] A. de Paz, A. Chotia, E. Maréchal, P. Pedri, L. Vernac, O. Gorceix, and B. Laburthe-Tolra, Phys. Rev. A 87, 051609 (2013).

[4] K. Aikawa, A. Frisch, M. Mark, S. Baier, A. Rietzler, R. Grimm, and F. Ferlaino, 
Phys. Rev. Lett. 108, 210401 (2012)

[5] M. Lu, N. Q. Burdick, S. H. Youn, and B. L. Lev, Phys. Rev. Lett. 107, 190401 (2011).

[6] Y. Tang, N. Q. Burdick, K. Baumann, and B. L. Lev, New J. Phys. 17, 045006 (2015).

[7] K. Aikawa, A. Frisch, M. Mark, S. Baier, R. Grimm, and F. Ferlaino, Phys. Rev. Lett. 112, 010404 (2014)

[8] T. Lahaye, C. Menotti, L. Santos, M. Lewenstein, and T. Pfau, Rep. Prog. Phys. 72, 126401 (2009).

[9] T. Lahaye, T. Koch, B. Fröhlich, M. Fattori, J. Metz, A. Griesmaier, S. Giovanazzi, and T. Pfau, Nature 448, 672 (2007)

[10] H. Kadau, M. Schmitt, M. Wenzel, C. Wink, T. Maier, I. Ferrier-Barbut, and T. Pfau, Nature 530, 194 (2016)

[11] R. N. Bisset and P. B. Blakie, Phys. Rev. A 92, 061603 (2015)

[12] K.-T. Xi and H. Saito, Phys. Rev. A 93, 011604 (2016).

[13] B. Naylor, M. Brewczyk, M. Gajda, O. Gorceix, E. Maréchal, L. Vernac, and B. Laburthe-Tolra, Phys. Rev. Lett. 117, 185302 (2016).

[14] S. Yi and H. Pu, Phys. Rev. A 73, 061602 (2006)

[15] D. H. J. O'Dell and C. Eberlein, Phys. Rev. A 75, 013604 (2007)

[16] R. M. Wilson, S. Ronen, J. L. Bohn, and H. Pu, Phys. Rev. Lett. 100, 245302 (2008).

[17] M. Klawunn, R. Nath, P. Pedri, and L. Santos, Phys. Rev. Lett. 100, 240403 (2008).

[18] M. Abad, M. Guilleumas, R. Mayol, M. Pi, and D. M. Jezek, Phys. Rev. A 79, 063622 (2009)

[19] M. Klawunn and L. Santos, New J. Phys. 11, 055012 (2009).

[20] Y. Kawaguchi and M. Ueda, Phys. Rep. 520, 253 (2012)

[21] S.-K. Yip, Phys. Rev. Lett. 83, 4677 (1999)

[22] T. Mizushima, K. Machida, and T. Kita, Phys. Rev. A 66, 053610 (2002)

[23] T. Mizushima, K. Machida, and T. Kita, Phys. Rev. Lett. 89, 030401 (2002)

[24] J.-P. Martikainen, A. Collin, and K.-A. Suominen, Phys. Rev. A 66, 053604 (2002)

[25] H. Saito, Y. Kawaguchi, and M. Ueda, Phys. Rev. Lett. 96, 065302 (2006)

[26] A.-C. Ji, W. M. Liu, J. L. Song, and F. Zhou, Phys. Rev. Lett. 101, 010402 (2008).

[27] J. Lovegrove, M. O. Borgh, and J. Ruostekoski, Phys. Rev. A 86, 013613 (2012).

[28] S. Kobayashi, Y. Kawaguchi, M. Nitta, and M. Ueda, Phys. Rev. A 86, 023612 (2012).

[29] M. O. Borgh and J. Ruostekoski, Phys. Rev. Lett. 109, 015302 (2012).

[30] J. Lovegrove, M. O. Borgh, and J. Ruostekoski, Phys. Rev. Lett. 112, 075301 (2014).

[31] J. Lovegrove, M. O. Borgh, and J. Ruostekoski, Phys. Rev. A 93, 033633 (2016)

[32] M. O. Borgh and J. Ruostekoski, Phys. Rev. Lett. 117, 275302 (2016).

[33] A. E. Leanhardt, Y. Shin, D. Kielpinski, D. E. Pritchard, and W. Ketterle, Phys. Rev. Lett. 90, 140403 (2003)

[34] L. S. Leslie, A. Hansen, K. C. Wright, B. M. Deutsch, and N. P. Bigelow, Phys. Rev. Lett. 103, 250401 (2009)

[35] J.-y. Choi, W. J. Kwon, and Y.-i. Shin, Phys. Rev. Lett. 108, 035301 (2012).

[36] J.-y. Choi, W. J. Kwon, M. Lee, H. Jeong, K. An, and Y.-i. Shin, New J. Phys. 14, 053013 (2012).

[37] M. W. Ray, E. Ruokokoski, S. Kandel, M. Möttönen, and D. S. Hall, Nature 505, 657 (2014)

[38] M. W. Ray, E. Ruokokoski, K. Tiurev, M. Möttönen, and D. S. Hall, Science 348, 544 (2015)

[39] D. S. Hall, M. W. Ray, K. Tiurev, E. Ruokokoski, A. H. Gheorghe, and M. Möttönen, Nat. Phys. 12, 478 (2016).

[40] S. W. Seo, S. Kang, W. J. Kwon, and Y.-i. Shin, Phys. Rev. Lett. 115, 015301 (2015)

[41] Y. Kawaguchi, H. Saito, and M. Ueda, Phys. Rev. Lett. 98, 110406 (2007).

[42] Y. Kawaguchi, H. Saito, K. Kudo, and M. Ueda, Phys. Rev. A 82, 043627 (2010).

[43] J. A. M. Huhtamäki and P. Kuopanportti, Phys. Rev. A 82, 053616 (2010).

[44] M. Vengalattore, S. R. Leslie, J. Guzman, and D. M. Stamper-Kurn, Phys. Rev. Lett. 100, 170403 (2008).

[45] Y. Eto, H. Saito, and T. Hirano, Phys. Rev. Lett. 112, 185301 (2014)

[46] L. Santos and T. Pfau, Phys. Rev. Lett. 96, 190404 (2006).

[47] Y. Kawaguchi, H. Saito, and M. Ueda, Phys. Rev. Lett. 96, 080405 (2006).

[48] K. Gawryluk, K. Bongs, and M. Brewczyk, Phys. Rev. Lett. 106, 140403 (2011)

[49] T. Świsłocki, T. Sowiński, J. Pietraszewicz, M. Brewczyk, M. Lewenstein, J. Zakrzewski, and M. Gajda, Phys. Rev. A 83, 063617 (2011).

[50] S. Yi and H. Pu, Phys. Rev. Lett. 97, 020401 (2006)

[51] Y. Kawaguchi, H. Saito, and M. Ueda, Phys. Rev. Lett. 97, 130404 (2006).

[52] M. Takahashi, S. Ghosh, T. Mizushima, and K. Machida, Phys. Rev. Lett. 98, 260403 (2007).

[53] T. P. Simula, J. A. M. Huhtamäki, M. Takahashi, T. Mizushima, and K. Machida, J. Phys. Soc. Jpn 80, 013001 (2011).

[54] F. K. Fatemi, K. M. Jones, and P. D. Lett, Phys. Rev. Lett. 85, 4462 (2000)

[55] D. J. Papoular, G. V. Shlyapnikov, and J. Dalibard, Phys. Rev. A 81, 041603 (2010).

[56] J. Ruostekoski and J. R. Anglin, Phys. Rev. Lett. 91, 190402 (2003).

[57] S. Yi, L. You, and H. Pu, Phys. Rev. Lett. 93, 040403 (2004).

[58] S. Ronen, D. C. E. Bortolotti, and J. L. Bohn, Phys. Rev. A 74, 013623 (2006)

[59] P. B. Blakie, C. Ticknor, A. S. Bradley, A. M. Martin, M. J. Davis, and Y. Kawaguchi, Phys. Rev. E 80, 016703 (2009).

[60] R. B. Diener and T.-L. Ho, Phys. Rev. Lett. 96, 190405 (2006).

[61] Y. Kawaguchi and M. Ueda, Phys. Rev. A 84, 053616 (2011).

[62] J. Werner, A. Griesmaier, S. Hensler, J. Stuhler, T. Pfau, A. Simoni, and E. Tiesinga, Phys. Rev. Lett. 94, 183201 (2005).

[63] B. Pasquiou, G. Bismut, Q. Beaufils, A. Crubellier, E. Maréchal, P. Pedri, L. Vernac, O. Gorceix, and B. Laburthe-Tolra, Phys. Rev. A 81, 042716 (2010).

[64] A. de Paz, B. Naylor, J. Huckans, A. Carrance, O. Gorceix, E. Maréchal, P. Pedri, B. Laburthe-Tolra, and L. Vernac, Phys. Rev. A 90, 043607 (2014).

[65] W. H. Press, S. A. Teukolsky, W. T. Vetterling, and B. P. Flannery, Numerical Recipes, 3rd ed. (Cambridge University Press, 2007). 
[66] E. N. Bulgakov and A. F. Sadreev, Phys. Rev. Lett. 90, 200401 (2003)

[67] B. C. Mulkerin, R. M. W. van Bijnen, D. H. J. O'Dell, A. M. Martin, and N. G. Parker, Phys. Rev. Lett. 111, 170402 (2013).

[68] S.-K. Yip, Phys. Rev. A 75, 023625 (2007)

[69] R. Barnett, A. Turner, and E. Demler, Phys. Rev. A 76, 013605 (2007)

[70] M. Kobayashi, Y. Kawaguchi, M. Nitta, and M. Ueda, Phys. Rev. Lett. 103, 115301 (2009).

[71] K. Tiurev, E. Ruokokoski, H. Mäkelä, D. S. Hall, and M. Möttönen, Phys. Rev. A 93, 033638 (2016)
[72] C. M. Savage and J. Ruostekoski, Phys. Rev. Lett. 91, 010403 (2003).

[73] J. D. Jackson, Classical Electrodynamics, 3rd ed. (Wiley, 1999).

[74] Note that while Eq. (A4) is here automatically fulfilled when the magnetic dipole field is written on the form of Eq. A7), the same is not true for the corresponding equations in the case of an electric dipole. In that case, a $\delta$-function correction to the electric-field analogs of Eqs. A7 and (A8) is necessary.

[75] S. L. Cornish, N. R. Claussen, J. L. Roberts, E. A. Cornell, and C. E. Wieman, Phys. Rev. Lett. 85, 1795 (2000). 